\title{
Electricity generation from the mud by using microbial fuel cell
}

\author{
Sitinoor Adeib Idris, Farah Nasyitah Esat, Ain Adilla Abd Rahim, W. A. Zahin Rizzqi W. Ruzlee and W. M. Zyaid Razali \\ Faculty of Chemical Engineering, Universiti Teknologi MARA, Johor, Pasir Gudang Campus
}

\begin{abstract}
Microbial fuel cells (MFCs) is a bio-electrochemical device that harnesses the power of respiring microbes to convert organic substrates directly into electrical energy. This is achieved when bacteria transfer electrons to an electrode rather than directly to an electron acceptor. Their technical feasibility has recently been proven and there is great enthusiasm in the scientific community that MFCs could provide a source of "green electricity". Microbial fuel cells work by allowing bacteria to do what they do best, oxidize and reduce organic molecules. Bacterial respiration is basically one big redox reaction in which electrons are being moved around. The objective is to generate electricity throughout the biochemical process using chemical waste basically sludge, via microbial fuel cells. The methodology includes collecting sludge from different locations, set up microbial fuel cells with the aid of salt bridge and observing the results in voltage measurement. The microbial fuel cells consist of two chambers, iron electrodes, copper wire, air pump (to increase the efficiency of electron transfer), water, sludge and salt bridge. After several observations, it is seen that this MFC can achieve up until 202 milivolts ( 0.202 volts) with the presence of air pump. It is proven through the experiments that sludge from different locations gives different results in term of the voltage measurement. This is basically because in different locations of sludge contain different type and amount of nutrients to provide the growth of bacteria. Apart from that, salt bridge also play an important role in order to transport the proton from cathode to anode. A longer salt bridge will give a higher voltage compared to a short salt bridge. On the other hand, the limitations that this experiment facing is the voltage that being produced did not last long as the bacteria activity slows down gradually and the voltage produced are not really great in amount. Lastly to conclude, microbial fuel cell essentially is a solution for a renewable energy emitted by bacteria activity that need to be take a further attention, research and development
\end{abstract}

\section{Introduction}

Microbial fuel cells (MFCs) use bacteria to convert the chemical energy of a particular substrate contained in wastewater into electrical energy. This is achieved when bacteria transfer electrons to an electrode rather than directly to an electron acceptor. Their technical feasibility has recently been proven and there is great enthusiasm in the scientific community that MFCs could provide a source of "green electricity" by exploiting domestic and industrial waste to generate power [1]-[3]. With the usage of mud which clearly contain bacteria in it, it is the main factor of the selection of mud for this microbial fuel cell. Bacteria need energy to survive, in the same way that humans need food to live. Bacteria get this energy in a two-step process. The first step requires the removal of electrons from some source of organic matter (oxidation), and the second step consists of giving those electrons to something that will accept them (reduction), such as oxygen or nitrate. If certain bacteria are grown under anaerobic conditions (without the presence of oxygen), they can transfer electrons to a carbon electrode (anode). The electrons then move across a wire under a load (resistor) to the cathode where they combine with protons and oxygen to form water. When these electrons flow from the anode to the cathode, they generate the current and voltage to make electricity [4]. Microbial fuel cells work by allowing bacteria to do what they do best, oxidize and reduce organic molecules. Bacterial respiration is basically one big redox reaction in which electrons are being moved around. Whenever a moving electrons present, the potential exists for harnessing an electromotive force to perform useful work [1].

As to assist the flow of electricity, the presence of salt bridge is crucial as it transports the proton in microbial fuel cell (MFC). In order to offset the very high cost of proton exchange membrane, salt bridges have been used in dual chamber MFCs [5]. The parameters that has been selected for the experimental procedure in this microbial fuel cell (MFC) are upon two factors which are; type of sludge and the length of salt bridge. It is expected that different type of sludge gives different result in term of the voltage in electricity. This is because from different location, it gives varies type of bacteria in the mud. The length of salt bridge also effects the generation of electricity because of the efficiency of transportation of proton [5].

The most immediately foreseeable application of an MFC is in waste water treatment. Microbes love sewage, and the conditions of a waste water treatment plant are 
ideal for the types of bacteria that can be used in an MFC. Exoelectrogens are more than happy to breakdown and metabolize the carbon rich sewage of a waste water stream to produce electrons that can stream into a cheap conductive carbon cloth anode. The electricity generated from the MFC also offsets the energy cost of operating the plant. As an added bonus, the bacteria eat a lot of the sludge normally present in waste water. One of the company that apply this process claims to be able to cut sludge down by $80 \%$ in their waste water treatment processes, which saves them time and money from having to transport sludge to a landfill or wasteland [1].

The application of MFC in integrated strategies towards sustainable bioenergy generation is current interest. MFCs are specifically designed to harness bioelectricity from benthic aquatic eco-system using natural habitats wherein, ecological water bodies are considered as a potential source to convert the organic rich sediment to energy. Plant based MFCs helps in the utilization of solar radiation to generate bioelectricity by integrating the rhizo deposits of living plant with electrodes. The scope of MFC application has been extended to the development of photo-electrocatalytic fuel cell using photosynthetic organisms as anodic biocatalyst to assess their potential to harness power [6].

The limitations of microbial fuel cell in this experiment is it only consists of two chambers and the amount of mud in each chamber which approximately $1.5 \mathrm{~L}$. The result that is predicted to be obtained is up to $200 \mathrm{mV}$. The performance is severely affected based on irreversible reactions and processes in the anode and the cathode compartments.

\section{Methodology}

\subsection{Materials}

Thick, soft, wet mud or a similar viscous mixture of liquid and solid components which contain polyphosphate bacteria is collected. The bacteria that present in this sludge carries out break down of nutrients and organic matter inside the sludge leads toward emission of electron which is particularly a contribution of small volts current. Two types of sludge from different location are collected as to determine the maximum performance of the sludge for the microbial fuel cell. First sample was collected at the bottom of the lakeside while the second sample was collected at the creek also from lakeside. The mud that are collected had different physical traits such as the colour and the roughness. Two containers were needed as the chamber of the microbial fuel cell. Both lids of containers were drilled for the insertion of copper wire that been attached with iron mesh electrode. Next, the wall of the containers were also drilled a hole each, for salt bridge connection. The salt bridge was prepared basically from these materials; cotton rope, salt and water. An amount of $98 \mathrm{~g}$ of salt was firstly being dissolved in water before it is heated for approximately 2 minutes. The cotton rope is soaked into the salt water for a period until the salt water has fully absorbed by the cotton rope. Salt bridge is a medium for proton to be flown from water filled container while electron counter flow by the copper wire. The electrode that were used in this experiment is iron mesh gauze as it is a good electricity conductor. The iron mesh were folded a few times and they were bind together to the both end of the copper wire that connect the two chambers. The iron electrode functions to attract electron release by the bacteria and flow it to copper wire.

\subsection{Procedure}

First, one of the container was filled with water and another container was filled with sludge. Then, the iron electrode were submerged into both of the container which contained water and sludge. Next, the lids of the container were closed and the aerobic bacteria container should be exposed to small amount of oxygen. After that, the copper wire at the both container were attached to alligator clips, then the alligator clips were clamped to the multimeter probes. The black probes should be connected to the electrode at the sludge where the electron emitted from the sludge were attracted by the anode. The current flow from the sludge container were detected and measured by the multimeter in volt measurement. Then, an air pump was turn on to increase the efficiency of electron transfer. Fig. 1 shows the diagram of how the microbial fuel cell was set up.

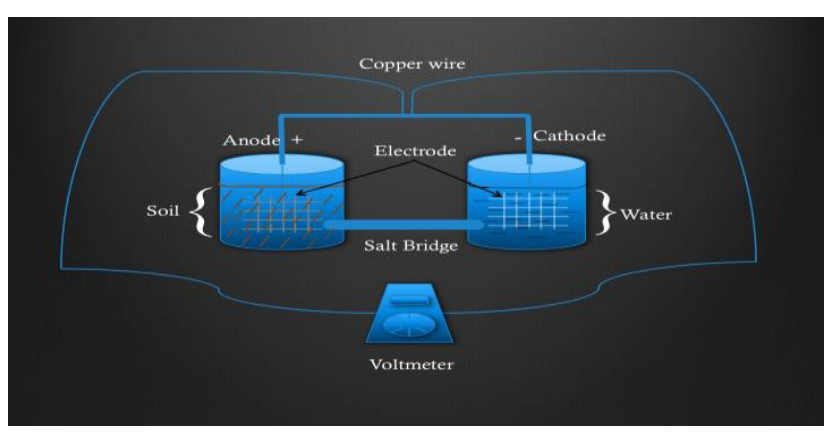

Figure 1. The set up of microbial fuel cell

\section{Results and discussion}

There were two parameters recorded as to determine the effect of each parameter towards the electrical charges produces from microbial fuel cell which are, location or type of sludge and length of salt bridge. Apart from that, the presence of air pump in the microbial fuel cell also give a huge impacts to the results obtain. Air pump was used as to increase the efficiency of the electrical charges produce from bacteria as it will supply more oxygen to the biotic ecosystem. Bacteria also known as aerobic which need oxygen to yield energy for growth. Different location of sludge will provide different amount of electrical charges form as in each sludge it contains different amount and type of nutrient. Fig. 2 and Fig. 3 shows the results which proves the difference in each sludge and length of salt bridge with the presence of air pump give different electrical measure in millivolt $(\mathrm{mV})$. 


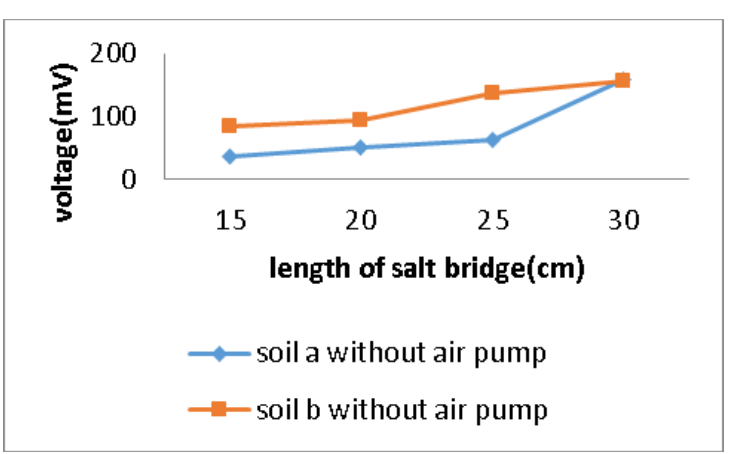

Figure 2. Electrical charges $(\mathrm{mV})$ to the length of salt bridge $(\mathrm{cm})$

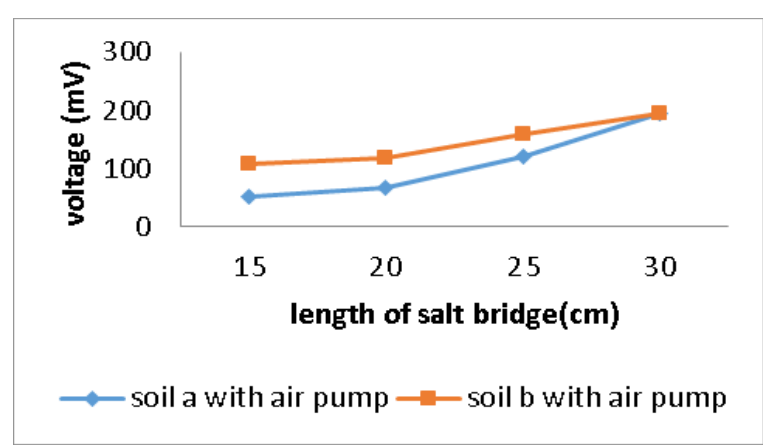

Figure 3. Electrical charges $(\mathrm{mV})$ to the length of salt bridge $(\mathrm{cm})$

Fig. 2 shows that sludge B produced higher amount of electrical charges rather than sludge A in every length of salt bridge. This results are proven that it follows the theory as the maximum amount of electrical charges can be convert is $789 \mathrm{mV}$ as it's depends on the substrate use in the experiment [7], [8]. The presence of added oxygen air pump also gives out a lot of effects towards the microorganism performances. Based on the Fig. 2 and 3 it shows a lot of differences in the electrical charges detected by the multimeter. It was proved that bacteria need more oxygen as energy to growth for their development. Thus from the reaction between the microorganism, an electrical charges will be form [1]. Sludge A was collected in different location as well as sludge B. This different location parameter will gives out different amount of microorganism or bacteria contain in each sludge.

Each sludge in different location will have different type of microorganism contain in it thus every type of microorganism will give different performance to the microbial fuel cell. In a review it stated that, axenic culture (single bacterial species) such as Proteus vulgaris will give out $0.8 \mathrm{~mA}$ while Rhodoferax ferrireducens from the same species will give out only $0.2 \mathrm{~mA}$ for the performances [7]. Microbial fuel cell were operated by using the concept of microorganism as the catalyst. This concept were shown practically in the waste water treatment plant. In waste water treatment plant, a specific type of microorganism will be supply to the plant with oxygen to improve the effectiveness of the process. As different microorganism will provide different performances, the pathway or the type of electrode connected to the substrate also will affect the microorganism performance [8].

The absence of any acceptors in an MFC makes some microorganisms pass the electrons onto the anode surface. Difference in redox potentials (i.e., the ability of a compound to donate or accept electrons, denoted $E_{o}$ and measured in volts) between the electron donor and the electron acceptor is the determinant of the potential energy available to the microorganism for anabolic processes. In an MFC the electrochemical redox potential difference of the anode and cathode determines how much energy is available and thus, generate the electricity [5]. By the results from the experiment, it is proven how redox reaction actually works with the presence of anode and cathode, by the assist of proton-transport which is the salt bridge.

The salt-bridge acts a proton transferring channel and this study aims to optimise the concentration of sludge that facilitates the maximum movement of the protons towards the cathode that corresponds with higher current generation efficiency of the MFC [3]. Therefore, the presence of salt bridge is crucial in order to ensure the transfer of protons work efficiently and eventually gives high electricity generation. After several experiments, it shows that different length of salt bridge give different results in terms of voltage. As well as shown in the Fig. 2 and 3 , although different types of sludge were used during the experiment, it still give the highest electrical charges for both sludge when the highest length of salt bridge were use which is $30 \mathrm{~cm}$ long.

The concentration of the salt bridge were recorded as constants as it shows that length of salt bridge can give huge effect toward the microbial fuel cell performances. Salt bridge were used to transfer the electron from cathode to anode as the longer the salt bridge, the higher the amount of electron will be transferred as it is proven during the experiments. The salt bridge with the length of $30 \mathrm{~cm}$ in the presence of air pump gives out $190.5 \mathrm{mV}$ for sludge A and $192.7 \mathrm{mV}$ for sludge B while the microbial fuel cell with $15 \mathrm{~cm}$ length of salt bridges gives out only 53.6mV for sludge A and 108.7 for sludge B.

The uses of microbial fuel cell in the waste water treatment offer several advantages such as cells are able to recover the chemical energy present in wastewater and turn it into electrical power [1]. This shows that the microorganism in the cell will react to convert chemical energy to the electrical energy. The sludge bacteria eat what is in the sludge, such as microscopic nutrients and sugars, and in turn, produce electrons that are released back into the sludge. Electrons are subatomic particles that have a negative charge which is used to create electricity, which is a form of energy. Most of the microorganism in microbial fuel cell depends on the oxogeneous or endogeneous mediator molecules transfer but a few can deliver electrons directly to the anode [4]. As in the experiment, which is using biofilm as the factor, microorganism can transfer electrons directly from the cell envelope to the electrode or across the biofilm.

The two chambers were stuck together tightly to prevent the salt bridge from getting loosed or deattached from the chamber. This is because the cotton rope that is used as salt bridge as it is easily deattached from the 
chamber when one of the chamber is moved and leakage happen. Thus, it will affect the result. Besides that, the used of the electrodes that have high surface area to increase the efficiency of the transfer of electron from the anode to the cathode. For example, in this experiment, the electrode that have $1 \mathrm{~cm}$ width is used. Therefore, by increasing the width to $2 \mathrm{~cm}$ is good enough to increase the voltage generated. The amount of power that is generated in these systems is affected by the surface area of the cathode relative to that of the anode and the surface of the membrane. For example, add more air pump to supply oxygen in cathode chamber. This can help in increasing the oxygen quantity in the water.

\section{Conclusions and recommendations}

The highest result for microbial fuel cell with the absence of air pump is $153 \mathrm{mV}$. The difference shows that the higher amount of oxygen provided will produce a result that is more efficient. This microbial fuel cell's experiment that convert microorganisms activity inside mud into electricity by redox reaction was predetermined as one of a green solution as it have a possibility to instill a non-harmful energy and environmentally friendly. Systems beneath the microbial fuel cell would give out an extensive search of ways to operate the bioelectrochemical device in more efficient and in high energy production. This fuel cell are feasible with the water treatment process in harvesting energy utilized by anaerobic digestion as a method used in the microbial fuel cell to collect bioenergy from wastewater, from this scope, MFC had clearly give an advantages in treating wastewater linearly producing electricity.

The microbial fuel cell experiment that had been carried out had given a process on learning the flaws of the device and the data that been acquired help to improvise a small scale of microbial fuel cell to reach its optimal condition. Currently, the size of MFC's is limited by the fact that electron transport only occurs in a bacteria layer immediately in contact with the electrodes. So while MFC's would have been a success in large scale batch processing of waste water streams, their true potential actually lies in small scale devices where the surface to volume ratio is high. There exists an optimal flow rate of reactants for increasing the voltage output of an MFC. Advances in microfluidics will allow engineers to make increasingly smaller MFC devices that can take advantage of this high surface to volume ratio. Research into advanced microfluidics, bacterial strains, a more robust separator membranes and efficient electrodes are the key to unlocking the maximum potential of MFCs.

The power density produced by these systems is typically limited by high internal resistance and electrode-based losses. When comparing power produced by these systems, it makes the most sense to compare them on the basis of equally sized anodes, cathodes, and membranes. In term of internal resistance of the MFC, it is dependent on both the resistance of the electrolyte between the electrodes and by the membrane resistance.
For optimal operation, anode and cathode need to be as close together as possible. Also proton migration significantly influences resistance-related losses [9-11].

\section{References}

1. F. J. Hernández-Fernández, Pérez de los Ríos, a., M. J. Salar-García, V. M. Ortiz-Martínez, L. J. LozanoBlanco, C. Godínez, J. Quesada-Medina, Recent progress and perspectives in microbial fuel cells for bioenergy generation and wastewater treatment. Fuel Processing Technology, 138, 284-297, (2015).

2. Y. Li, Y. Wu,B. Liu,H. Luan, T. Vadas, W. Guo. B. Li, Bioresource Technology Self-sustained reduction of multiple metals in a microbial fuel cell - microbial electrolysis cell hybrid system. BIORESOURCE TECHNOLOGY, 192, 238-246. (2015).

3. W. Liu, S. Cheng, D. Sun, H. Huang, J. Chen, \& K. Cen, Inhibition of microbial growth on air cathodes of single chamber microbial fuel cells by incorporating enrofloxacin into the catalyst layer. Biosensors \& Bioelectronics, 72, 44-50. (2015).

4. B. E. Logan, Microbial Fuel Cells. Retrieved from www.research.psu.edu/.../MFC_QandA.pdf, (2008).

5. T. R. Sreekrishnan and S. Sevda, Effect of salt concentration and mediators in salt bridge microbial fuel cell for electricity generation from synthetic wastewater. Retrieved from http://www.ncbi.nlm.nih.gov/pubmed/22423995, (2012).

6. S. V. Mohan, G. Velvizhi, J. A. Modestra \& S. Srikanth, Microbial fuel cell: Critical factors regulating bio-catalyzed electrochemical process and recent advancements. Renewable and Sustainable Energy Reviews, 40, 779-797. (2014).

7. K. Rabaey, \& W. Verstraete, Microbial fuel cells: Novel biotechnology for energy generation. Trends in Biotechnology, 23(6), 291-298. (2005).

8. P. Aelterman, K. Rabaey, H. T. Pham, N. Boon and, W. Verstraete, Continuous electricity generation at high voltages and currents using stacked microbial fuel cells, Environmental Science and Technology, 40 (10), 3388-3394, (2006).

9. H. Wang, H. Luo, P. H. Fallgren, S. Jin., \& Z. J. Ren, Bioelectrochemical system platform for sustainable environmental remediation and energy generation. Biotechnology Advances, 33(3-4), 317-334, (2015).

10. Y. Zhang,, Y. Wang., \& I. Angelidaki, Alternate switching between microbial fuel cell and microbial electrolysis cell operation as a new method to control $\mathrm{H}_{2} \mathrm{O}_{2}$ level in Bioelectro-Fenton system. Journal of Power Sources, 291, 108-116, (2015).

11. H. Rismani-Yazdi, Cathodic limitations in microbial fuel cells: An overview. Retrieved from http://www.sciencedirect.com/science/article/pii/S0 37877530800387X, (2008). 